\title{
The organic interlamellar layer in abalone nacre: formation and mechanical response
}

\author{
Maria Isabel López ${ }^{1}$ and Marc André Meyers ${ }^{1,2,3}$
}

(Tel.:858-204-4294; FAX: 858-534-5698; email: mameyers@ucsd.edu)

\author{
${ }^{1}$ Materials Science and Engineering Program, University of California, San Diego, La Jolla, USA \\ ${ }^{2}$ Department of Mechanical and Aerospace Engineering, University of California, San Diego, La \\ Jolla, USA \\ ${ }^{3}$ Department of Nanoengineering, University of California, San Diego, La Jolla, USA
}

\begin{abstract}
The interlamellar organic layer plays a key role in establishing the tensile mechanical response of nacre, while changing the compressive response in only a marginal manner. We conduct observations on the epithelial layer of the abalone foot in direct contact with the extrapallial layer where the deposition process takes place and identify cilia, microvilli, and secretory cells which determine the deposition of chitin to form the interlamellar organic layer. On the basis of these observations we propose a mechanism for the deposition of interlamellar organic layers.
\end{abstract}

We show that the fraction of pores, as well as their diameter grow rapidly as the interlamellar layer is extended uniaxially or biaxially, and compare these calculations with the observed values. In the calculations we assume a Poisson's ratio equal to zero in the plane of the lamellae. This assumption is justified by the thickness of the organic layer $(20-50 \mathrm{~nm})$ being equal to twice the diameter of the chitin fibrils; consequently the expansion of the membrane occurs at a constant thickness. As an illustration of this effect, an externally applied strain of 0.5 increases 
the initial pore diameter (typically equal to $20-50 \mathrm{~nm}$ ) to a value of ten times $(0.2-0.5 \mu \mathrm{m})$. These calculations explain the observations of large pores reported in the literature and interpret them as the result of externally applied loads.

\section{Introduction}

The nacreous component of abalone, nautilus, and other calcium carbonate based shells is composed of tablets of aragonite separated by organic layers. The high toughness (in comparison with monolithic $\mathrm{CaCO}_{3}$ ) is attributed to the synergy between organic and mineral components. Foremost, the inner nacreous layer of the abalone shell, Haliotis rufescens, is a mineral/ organic composite (95 wt $\%$ calcium carbonate, $5 \mathrm{wt} \%$ organic material) made up of nanoscale tablets of aragonite $\left(\mathrm{a} \mathrm{CaCO}_{3}\right.$ polymorph) separated by an interlamellar matrix of organic biopolymers, described as a "brick-and mortar" structure [1-6]. Mineral bridges and the their interlocking action are important mechanisms of toughening of nacre $[7,8]$.

The mineral tiles are typically $0.4-0.6 \mu \mathrm{m}$ thick and 8-10 $\mu \mathrm{m}$ wide and the organic interlamellar layers range from 20 to $50 \mathrm{~nm}$ thick. The outside layer of the shell is calcitic, while the inside is aragonitic. The epithelial layer of the abalone foot (Figure 1a) plays an important role in the growth of the shell. Figure $1 \mathrm{~b}$ shows the fracture surface; the tablets are organized in stacks, the result of the growth process in the "Christmas tree" or "terraced cone" arrangement. This highly ordered structure results in outstanding mechanical properties that have been intensely investigated experimentally, analytically, and computationally [2, 3, 8-21].

Many theories on the crystal nucleation and growth of abalone nacre have been advanced, including some stating that the crystallization happens within preformed organic matrix compartments [13, 22-24]. Two of them are: (a) heteroepitaxial capping (a termination of the 
crystal growth, followed by heteroepitaxial nucleation of the next crystal layer) [25]; (b) quantized secretion and sequential crystallization (a layer by layer formation of the organic matrix and mineral crystals) $[18,26]$. These theories propose that each crystal layer is nucleated on the organic matrix.

Other models propose that nacre is not formed by a direct nucleation of each layer on the organic matrix, but through a continuous growth of the aragonite crystals from layer to layer through holes or pores existing in the interlamellar organic layers [8, 14, 26-28]. Characterization by Belcher and Gooch [9, 29], Checa et al. [30], and Lin et al. [11] suggests that the tablets present in nacre belonging to the same stack ("Christmas tree") have the same orientation. These studies propose that the holes in the interlamellar layer serve as conduits for continuous growth from one layer to the next.

There have been a number of studies on both nacreous and calcitic components in shells [e.g., 31-33]. The nacre is much tougher because of its brick-and-mortar microstructure, whereas the calcite has a higher hardness (nanoindentation equal to $\sim 5 \mathrm{GPa}$ vs. $\sim 3 \mathrm{GPa}$ for nacre) but is more brittle. The calcite is only the external layer and for the shell investigated here, Figure 1, its thickness is approximately equal to that of the aragonitic nacre.

There are computational studies on the nanoasperities [34] and the elastic response of the organic phase and how it is influenced by the proximity of the mineral $[35,36]$. The current paper sheds new light on how the organic nanolayer changes with biaxial deformation.

\section{Experimental Techniques}

Demineralization was performed by sectioning slices of nacre from the growing edge of fresh abalone shells, washed in deionized water, and placed in $0.6 \mathrm{M}$ EDTA, $\mathrm{pH} 8.0$ for two 
weeks at $20{ }^{\circ} \mathrm{C}$. They were then gradually dehydrated in a series of $25 \%, 50 \%, 75 \%$, and $100 \%$ ethanol for periods exceeding 40 minutes at each stage. For the observation in the scanning electron microscope, sections were then critical point dried in order to preserve the structure.

For transmission electron microscopy observation, the outer surface of the epithelium (which is in contact with the growth edge of the shell) was cut into $1 \mathrm{~mm}$ thick sections. A primary fixation was performed by immersing the tissue sections in $2.5 \%$ paraformaldehyde, 2.5\% glutaraldehyde in $0.1 \mathrm{M}$ cacodylate buffer, $\mathrm{pH} 7.4$ for 2 hours in ice. Primary washing of the specimens was done 5 times in $0.15 \mathrm{M}$ cacodylate buffer (with a 3 minute duration in between). Then a secondary fixation was done by placing the specimens in $1 \%$ osmium tetroxide in $0.15 \mathrm{M}$ cacodylate buffer, $\mathrm{pH} 7.4$ for 2 hours in ice. A secondary washing was done three times with 5 minute duration in between in double distilled water. Then the specimens where stained in $1 \%$ uranyl acetate in $25 \%$ ethanol and incubated at $8^{\circ} \mathrm{C}$ overnight.

The specimens where then washed again with double distilled water 5 times; in the final wash the specimens were allowed to sit in the double distilled water 5 minutes. Dehydration was then performed by immersing the specimens in a series of graded ethanol solutions $(20 \%, 30 \%$, $50 \%, 70 \%, 90 \%)$, each for $10 \mathrm{~min}$, and then in 100\% ethanol for three times (10 min/each time). The specimens were then infiltrated with Spurr's resin by being placed in a 33/67, 50/50, 67/33, $75 / 25$ Spurr's resin/ethanol, and 3 X 100\% Spurr's resin solution (placed for 24 hours each exchange). Polymerization was performed by placing specimens in an aluminum tray in a $60^{\circ} \mathrm{C}$ oven for 24 hours. The embedded tissue was cut into thin sections ( $70-90 \mathrm{~nm}$ thick) by a glass knife in an ultramicrotome. Ultramicrotomed sections were then placed on a copper grid for TEM observation, which was conducted in a FEI Sphera microscope at an accelerating voltage of $200 \mathrm{kV}$. 


\section{Results and Discussion}

\subsection{Formation of Interlamellar Layers}

Although nacre has been subjected to a large number of investigations with the purpose of elucidating the toughness mechanisms and with the ultimate goal of creating bioinspired composites, the organic interlamellar layers have received much less attention. This organic matrix consists of $\beta$-chitin fibrils of $\sim 20 \mathrm{~nm}$ diameter surrounded by acidic macromolecules that attach themselves to the top and bottom surface of the tiles [37, 38].

Figure 2 shows a perspective view of the several organic interlayers in demineralized nacre. The calcium carbonate tablets, seen in Figure 1b, are completely dissolved, revealing successive layers, numbered from 1 to 12 . It can be seen that these layers contain holes.

The organic interlayer appears to be periodically deposited (every $\sim 0.4-0.6 \mu \mathrm{m}$ ) by the epithelial layer of the animal. In abalone shell, two different polymorphs exist within the shell (Figure 1a): the outside (under the Periostracum) is calcitic, whereas the inside is aragonitic. These sections are formed by a precise control of the deposition of the organic constituent by the outer epithelium. The cells involved in movement of mineral ions to the site of deposition and in the secretion of organic matter that will become the matrix of the deposit [39] are shown in Figure 3. In mollusks, ion movement is bidirectional and occurs through six compartments: the outer medium, body epithelium, blood and tissues, mantle epithelium, extrapallial fluid, and finally the shell. The ions are provided from the environment and from the animal's metabolism. Figure 3 (a-c) shows TEM micrographs of thin sections taken from the epithelial tissue. The secretory cells and subepithelial glands commonly contain various types of glycoconjugates that can be specifically detected performing a histochemistry study, which is beyond the spectrum of this work. However, mucoid substances such as glycoproteins and mucopolysaccharids do not 
have a high affinity to electron microscopic staining agents; thus, those vesicles will appear less electron-dense in the transmission electron micrographs. The epithelium is typically lined by columnar epithelial cells (that range between 4 to $5 \mu \mathrm{m}$ in length) seated on the basal lamina interspersed with ciliated cells (Figure 3d) and different types of secretory cells (Figure 3c) embedded in the subepithelial space. Figure $3 \mathrm{e}$ is a schematic that represents the edge of the mantle showing the cilia interspaced by microvilli. Although the specific functions of the structure are debated, observation of microvilli suggests that there is a secretion of substances responsible for construction of the organic interlamellar layer. It is possible that the cilia have a sensing function and regulate the production and subsequent deposition of chitin on the growth surface. Figure 4 shows a schematic illustrating how the deposition of the organic interlamellar layer is thought to occur. Secretory cells generate the chitin fibrils which are deposited periodically. The microvilli and cilia 'probe' the nacre surface and control the deposition of the interlamellar layer.

Mineral ion transport is allowed through existing holes that have an approximate diameter of 20-60 nm (Figure 5a). Due to the permeability of the membrane to the $\mathrm{Ca}^{2+}$ and $\mathrm{CO}_{2}^{-3}$ ions, they flow through holes and lateral growth can continue on embedded tablets allowing continuous growth between the mineral layers by the formation of mineral nano-bridges $[8,12,14]$.

The main experimental support for this model is that: (a) tiles remain atomically coherent and monocrystalline from layer to layer; (b) the interlamellar organic matrix has been significantly studied [18] and the pores have been observed by several groups [8, 12, 14, 40-44]. By this model, the pores control the position of the bridges that enable tablet growth and thus the 
aragonite grows as a single crystal maintaining its crystallographic alignment in the c-, a- and baxes.

\subsection{Growth of Nacre through the Organic Interlamellar Layers}

The organic interlayer in nacre has been the subject of a considerable number of structural and mechanical investigations [11, 14, 42, 43, 45-48]. Meyers et al. [14] used nanoindentation and the sagging of the membrane to estimate its mechanical response, concluding the flow stress is very low in the wet condition while the membrane becomes stiff and brittle in the dry state. Bezares et al. 42, 43, 48] and López et al. [49] established the mechanical response of the organic layer in tension and found it to be highly strain-rate dependent. The slope of the stress-strain curve increased from 12.5 MPa to $115 \mathrm{MPa}$ when the strain rate was raised from $10^{-4} \mathrm{~s}^{-1}$ to $10^{-2} \mathrm{~s}^{-1}$,

An interesting observation that is highly relevant to both the growth mechanism of the nacre and the mechanical response is that the porous structure of the organic interlamellar layers is interspersed with holes of diameters between 20 and $50 \mathrm{~nm}$. These holes grow at a rate much larger than the applied strain. Belcher and Gooch [29] observed that the size of the holes increased significantly with stretching of the membrane. Additional observations by Meyers et al. [11] confirmed this and a qualitative argument was made based on the fact that the hoop stresses at the surface of the hole were twice the remote traction. The analytical treatment of membranes containing holes is well developed, the first efforts dating from the 1950's (Rivlin and Thomas [50]). More recently, Lubarda [51] presented a comprehensive constitutive analysis of the subject under in-plane deformation.

\subsection{Mechanical Analysis of Pore Size Evolution}


The objective of this section is to obtain quantitative analytical solutions for the growth of the hole as a function externally applied stretching and to compare these results with observations of the organic layer subjected to uniaxial tension. These calculations increase our understanding of the contribution of this interlamellar layer to the toughness of nacre.

A hexagonal arrangement of holes is considered (Figure 6a); from this arrangement we extract a single, separate representative areal element (Figure 6b) in an isoareal deformation, $\mathrm{A}_{0}=\mathrm{A}:$

$$
A_{0}=\pi b_{0}^{2}-\pi a_{0}^{2}=\pi b_{1} b_{2}-\pi a_{1} a_{2}=A
$$

$a_{0}$ and $b_{0}$ are the initial values of the inner and outer radius, respectively. The corresponding values after stretching are $a_{1}$ and $a_{2}, b_{1}$ and $b_{2}$. In the case where $b_{1}=b_{2}=b$ and $a_{1}=a_{2}=a$, we have: $b^{2}-b_{0}^{2}=a^{2}-a_{0}^{2}$. This is justified by the fact that the thickness of the layer, $20-50 \mathrm{~nm}$ is equal to twice the diameter of the chitin fibrils $(\sim 10-20 \mathrm{~nm})$. The thickness of the individual fibrils is not affected by the expansion, since they only slide by each other. Thus, the thickness of the layer is constant with extension and Poisson's ratio in plane is 0 .

The engineering strain is defined as:

$$
\varepsilon=\frac{b-b_{0}}{b_{0}}
$$

In planar isotropic deformation $\varepsilon_{11}=\varepsilon_{22}$ (biaxial stress with $\sigma_{11}=\sigma_{22}$ ). Substituting Equation 2 into Equation $b^{2}-b_{0}^{2}=a^{2}-a_{0}^{2}$ :

$$
\frac{a}{a_{0}}=\sqrt{\frac{b_{0}^{2}}{a_{0}^{2}} \varepsilon(\varepsilon+2)+1}
$$


The experimental observations of Figure 5 can be used to approximate values for the hole diameter $\left(2 a_{0}=40 \mathrm{~nm}\right)$. Figure 7 shows the plots of strain vs. $\frac{a}{a_{0}}$ for values $2 \leq \frac{b_{0}}{a_{0}} \leq 10$. The initial porosity is defined as $\left(\frac{a_{0}}{b_{0}}\right)^{2}$ and is shown in the same plot. It can be seen that for larger membrane-to-pore area ratios, pore expansion is highly sensitive to strain. Experimental observations (Figures 2 and 5) show that the initial membrane-to-pore size ratio ranges between $2 \leq \frac{b_{0}}{a_{0}} \leq 3$. Assuming that the organic interlayers are isotropic, a pore stretches to over 10 times the initial pore size when strain values of $\sim 10 \%$ are reached. Observations of the pore structure in the demineralized nacre (after the tensile load is applied, shown in Figure 5) show significant pore size increases. This was also observed by Belcher and Gooch [29], as mentioned earlier. Indeed, in some cases pores stretch beyond 10x the initial pore size.

\subsection{Radial and Hoop Stresses}

We consider the membrane to be under an external traction $T\left(\sigma_{11}=\sigma_{22}=T\right)$. Similarly to Figure $6 b$, the membrane has initial (undeformed) inner and outer radii of $a_{0}$ and $b_{0}$ (Figure 8). Let us consider an arbitrary point inside the membrane at a distance $r_{0}$. As traction is applied, $r_{0}$ changes to $r$. The radial and hoop stresses at $r$ can be calculated. The forces are as depicted in Figure 8. In order to determine the stresses inside the membrane as a function of its stretching, we need the equilibrium equation, a constitutive relationship, and the boundary conditions. The equilibrium equation in the radial direction is found from:

$$
\Sigma F_{r}=0
$$

$\sigma_{\mathrm{r}}$ and $\sigma_{\theta}$ are the radial and tangential stresses, respectively. The forces are: 


$$
\left(\sigma_{r 1}\right) r_{1} d \theta \text { and }-\left(\sigma_{r 3}\right) r_{3} d \theta
$$

Note that, in Figure 8, the radial stress changes with radius. The radial force components from the tangential stresses are:

$$
-\sigma_{\theta}\left(r_{1}-r_{3}\right) \sin \left(\frac{d \theta}{2}\right)
$$

Since $\mathrm{d} \theta$ is small, we can approximate: $\sin \frac{d \theta}{r}=\frac{d \theta}{r}$, and $r_{1}-r_{3}=d r$

The equilibrium equation (Eq. 4), predicts:

$$
\left(\sigma_{r 1}\right) r_{1} d \theta-\left(\sigma_{r 3}\right) r_{3} d \theta-2 \sigma_{\theta} d r\left(\frac{d \theta}{2}\right)=0
$$

Dividing by $d \theta \square d r$ :

$$
\frac{\left(\sigma_{r 1}\right) r_{1}-\left(\sigma_{r 3}\right) r_{3}}{d r}-\sigma_{\theta}=0
$$

Since:

$$
\frac{d\left(\sigma_{r} r\right)}{d r}=r \frac{d \sigma_{r}}{d r}+\sigma_{r}
$$

Thus,

$$
r \frac{d \sigma_{r}}{d r}+\sigma_{r}-\sigma_{\theta}=0
$$

This is the radial equation of equilibrium. 
We use a form of the Mooney-Rivlin for constitutive equation adopted by Lubarda [51] for a non-linear material. In radial coordinates it is in the form:

$$
\sigma_{r}-\sigma_{\theta}=\mu\left(\lambda_{\theta}^{-2}-\lambda_{\theta}^{2}\right)
$$

The principal hoop stretch is defined as:

$$
\lambda_{\theta}=\frac{r}{r_{0}}
$$

and $\mu$ is the shear modulus of the membrane.

$$
d r=r_{0} d \lambda_{\theta}+\lambda_{\theta} d r_{0}
$$

$$
d r_{0}=\lambda_{\theta} d r
$$

where the initial radius $r_{0}$ to an arbitrary point of the membrane becomes: $r=r\left(r_{0}\right)$. Using the expressions for $d r$ and $d r_{0}$ from above, one obtains:

$$
\frac{d \sigma_{r}}{d \lambda_{\theta}}=-\mu\left(\frac{1}{\lambda_{\theta}}+\frac{1}{\lambda_{\theta}^{3}}\right)
$$

Separating variables and integrating:

$$
\int d \sigma_{r}=\int-\mu\left(\frac{1}{\lambda_{\theta}}+\frac{1}{\lambda_{\theta}^{3}}\right) d \lambda_{\theta}
$$

Substituting $\lambda_{\theta}=\frac{r}{r_{0}}$ into Eqn. 12: 


$$
\sigma_{r}=\frac{1}{2} \mu\left(\frac{r_{0}^{2}}{r^{2}}-\ln \frac{r^{2}}{r_{0}^{2}}\right)+D
$$

Following Lubarda [51], we eliminate $r$ by making the substitution $r^{2}=r_{0}^{2}+C^{2}$, because $r$ changes with stress:

$$
\sigma_{r}=\frac{1}{2} \mu\left[\frac{1}{1+\frac{C^{2}}{r_{0}^{2}}}-\ln \left(1+\frac{C^{2}}{r_{0}^{2}}\right)\right]+D
$$

Similarly, the hoop stress follows from Equation 10:

$$
\sigma_{\theta}=\frac{1}{2} \mu\left[2\left(1+\frac{C^{2}}{r_{0}^{2}}\right)-\frac{1}{1+\frac{C}{r_{0}^{2}}}-\ln \left(1+\frac{C^{2}}{r_{0}^{2}}\right)\right]+D
$$

The boundary conditions are $\sigma=0$ when $r_{0}=a_{0}$ and $\sigma=T$ when $r_{0}=b_{0}$. Thus,

$$
\begin{aligned}
& 0=\frac{1}{2} \mu\left(\frac{a_{0}^{2}}{a_{0}^{2}+C}-\ln \frac{a_{0}^{2}+C}{a_{0}^{2}}\right)+D \\
& T=\frac{1}{2} \mu\left(\frac{b_{0}^{2}}{b_{0}^{2}+C}-\ln \frac{b_{0}^{2}+C}{b_{0}^{2}}\right)+D
\end{aligned}
$$

Substituting $D$ from Equation 16 into Equation 17 and dividing all terms by $a_{0}$ we solve numerically for $C$ and $D$ for $\frac{b_{0}}{a_{0}}=2,3$, and 4 (going beyond the experimental observations on the membrane to pore ratio of 2-3), after setting $T=0.5 \mu$. The normalized radial and hoop stresses are plotted versus $r_{0} / a_{0}$ in Figure 9. The radial stresses vary from zero, at the inner radius to $T$, 
the applied traction, at the external surface. The hoop stresses at the internal surfaces of the voids are considerably larger than the ones in the outside. The ratios of hoop stresses to the applied traction are $\sim 1.76, \sim 1.62$, and $\sim 1.59$ for porosities of $0.25,0.11$, and 0.0625 and $\frac{b_{0}}{a_{0}}=2,3$, and 4 , respectively. These results are in agreement with an earlier prediction by Meyers et al. [11].

\section{Conclusions}

The research whose results are reported herein reveals two important aspects about the interlamellar membrane in nacreous shells, pertaining to its formation and response to external stretching:

- The characterization of the epithelial layer reveals the presence of cilia, microvilli, and secretory cells which are the biological agents responsible for regulating the formation of the interlamellar organic layer, controlling its thickness and periodicity.

- We present an analysis of the thin (porous) interlamellar organic membrane, such as that present in abalone nacre. When the membrane-to-pore size ratio $\left(\mathrm{b}_{0} / \mathrm{a}_{0}\right)$ is large, the fractional increase in pore size is many times higher than externally applied strain. Thus, we explain the presence of pores that have increased from 20 to $200 \mathrm{~nm}$ in radius which are routinely seen in the organic layer. This requires that the Poisson's ratio in the plane be zero, consistent with the chitin fibrils retaining their thickness. The thickness of membrane does not change as it stretches.

- The radial and hoop stresses in the interlamellar layer are calculated as a function of radius $r$, for different porosities $(0.11,0.25$, and 0.0625$)$. The hoop stresses vary 
between $3 T$ and $5 T$ ( $T$ is the external traction) in the membrane, whereas the radial stresses increase from zero in the inside to close to $T$ in the outside.

\section{Acknowledgements}

This research was supported by a Ford Foundation Fellowship and by the National Science Foundation Grant DMR 1006931. We appreciate discussion on the topic of void expansion with Professor L.A. Lubarda. Help by Mason Mackey and Norm Olson are also appreciated for their training and support with the transmission electron microscopy. The abalone come from tanks at the Scripps Institute of Oceanography Laboratory at Hubbs Hall and we appreciate them being kept by Phil Zerofski.

\section{References}

[1] M. Sarikaya, Microsc Res Techniq, 27 (1994) 359-359.

[2] A. Lin, M.A. Meyers, Mat Sci Eng a-Struct, 390 (2005) 27-41.

[3] R. Menig, M.H. Meyers, M.A. Meyers, K.S. Vecchio, Acta Mater, 48 (2000) 2383-2398.

[4] F. Barthelat, C.M. Li, C. Comi, H.D. Espinosa, J Mater Res, 21 (2006) 1977-1986.

[5] X.D. Li, W.C. Chang, Y.J. Chao, R.Z. Wang, M. Chang, Nano Lett, 4 (2004) 613-617.

[6] F.D. Fleischli, M. Dietiker, C. Borgia, R. Spolenak, Acta Biomater, 4 (2008) 1694-1706.

[7] K.S. Katti, D.R. Katti, S.M. Pradhan, A. Bhosle. J Mater Res 20 (2005) 1097-1100.

[8] T.E. Schäffer, C. IonescuZanetti, R. Proksch, M. Fritz, D.A. Walters, N. Almqvist, C.M. Zaremba, A.M. Belcher, B.L. Smith, G.D. Stucky, D.E. Morse, P.K. Hansma, Chem Mater, 9 (1997) 1731-1740.

[9] M. Fritz, A.M. Belcher, M. Radmacher, D.A. Walters, P.K. Hansma, G.D. Stucky, D.E. Morse, S. Mann, Nature, 371 (1994) 49-51.

[10] C.M. Zaremba, A.M. Belcher, M. Fritz, Y.L. Li, S. Mann, P.K. Hansma, D.E. Morse, J.S. Speck, G.D. Stucky, Chem Mater, 8 (1996) 679-690.

[11] M.A. Meyers, A.Y.M. Lin, P.Y. Chen, J. Muyco, J Mech Behav Biomed, 1 (2008) 76-85.

[12] A.Y.M. Lin, P.Y. Chen, M.A. Meyers, Acta Biomater, 4 (2008) 131-138.

[13] G. Bevelander, H. Nakahara, Calc Tiss Res, 3 (1969) 84-\&.

[14] M.A. Meyers, C.T. Lim, A. Li, B.R.H. Nizam, E.P.S. Tan, Y. Seki, J. McKittrick, Mat Sci Eng C-Mater, 29 (2009) 2398-2410.

[15] M. Sarikaya, I.A. Aksay, Results Probl Cell Differ, 19 (1992) 1-26.

[16] N. Watabe, K.M. Wilbur, Nature, 188 (1960) 334-334.

[17] K. Wada, Bulletin of the Japanese Society for the Science of Fish, 9 (1964) 1087-1098.

[18] K.M. Towe, G.H. Hamilton, Calc Tiss Res, 1 (1968) 306-\&. 
[19] S. Manne, C.M. Zaremba, R. Giles, L. Huggins, D.A. Walters, A. Belcher, D.E. Morse, G.D. Stucky, J.M. Didymus, S. Mann, P.K. Hansma, P Roy Soc B-Biol Sci, 256 (1994) 17-23.

[20] G. Falini, S. Albeck, S. Weiner, L. Addadi, Science, 271 (1996) 67-69.

[21] L. Addadi, D. Joester, F. Nudelman, S. Weiner, Chem-Eur J, 12 (2006) 981-987.

[22] H. Nakahara, Venus Jpn. J. Malac., 38 (1979) 205-211.

[23] S.W. Wise, Science, 167 (1970) 1486-\&.

[24] S. Uozumi, Y. Togo, Jour. Fac. Sci., Hokkaido Univ., Ser. IV, 17 (1975) 153-172.

[25] D.E. Morse, M.A. Cariolou, G.D. Stucky, C.M. Zaremba, P.K. Hansma, in: Materials Research Society Symposium, 1993, pp. 59-67.

[26] N. Watabe, Prog Cryst Growth Ch, 4 (1981) 99-147.

[27] H. Mutvei, Scanning Electron Microsc. , 2 (1979) 457-462.

[28] K. Wada, Biomineralization 6(1972) 141-159.

[29] A.M. Belcher, E.E. Gooch, in: E. Bäuerlein (Ed.) Biomineralization: From Biology to Biotechnology and Medical Application., Wiley-Interscience, 2000, pp. 221.

[30] A.G. Checa, T. Okamoto, J. Ramirez, P Roy Soc B-Biol Sci, 273 (2006) 1329-1337.

[31] M.A. Meyers, P.Y. Chen, A.Y.M. Lin, Y. Seki. Prog Mater Sci 53(2008)1-206.

[32]P.Y. Chen, J. McKittrick, M.A. Meyers. Prog Mater Sci 57(2012)1492-1704.

[33] A. S. Schneider, B.Heiland, N. J.Peter, C.Guth, E.Arzt, \& I. M.Weiss. . BMC biophysics, 5(2012) 19.

[34] D. R. Katti, S. M. Pradhan, and K. S. Katti. Rev Adv Mater Sci 6(2004) 162-168.

[35] P. Ghosh, D.R. Katti, K.S. Katti. Biomacromolecules 8 (2007) 851-856.

[36] P. Ghosh, D.R. Katti, K.S. Katti. J Nanomater (2008) 8.

[37] M. Sarikaya, Gunnison, K. E., Yasrebi, M. and Aksay, I. A., Materials Research Society, 174 (1989) 109-166.

[38] Y. Levi-Kalisman, G. Falini, L. Addadi, S. Weiner, J Struct Biol, 135 (2001) 8-17.

[39] Saleuddi.As, K.M. Wilbur, Can J Zoolog, 47 (1969) 51-\&.

[40] P. Westbroek, E.W. De Jong, Biomineralization and biological metal accumulation : biological and geological perspectives : papers presented at the fourth International Symposium on Biomineralization, Renesse, the Netherlands, June 2-5, 1982, D. Reidel Pub. ; Sold and distributed in the U.S.A. and Canada by Kluwer Boston Inc., Dordrecht, Holland ; Boston Hingham, MA, 1983.

[41] H. Nakahara, G. Bevelander, M. Kakei, Venus (Jap. J. Malac), 41 (1982) 33-46.

[42] J. Bezares, R.J. Asaro, M. Hawley, J Struct Biol, 163 (2008) 61-75.

[43] J. Bezares, R.J. Asaro, M. Hawley, J Struct Biol, 170 (2010) 484-500.

[44] A.K. Dastjerdi, R. Rabiei, F. Barthelat, J Mech Behav Biomed, 19 (2012) 50-60.

[45] A.G. Checa, A.B. Rodriguez-Navarro, Biomaterials, 26 (2005) 1071-1079.

[46] J.H.E. Cartwright, A.G. Checa, J R Soc Interface, 4 (2007) 491-504.

[47] N. Yao, A.K. Epstein, W.W. Liu, F. Sauer, N. Yang, J R Soc Interface, 6 (2009) 367-376.

[48] J. Bezares, Z. Peng, R.J. Asaro, Q. Zhu, Journal of Theoretical Applied Mechanics, 38 (2011) 75-106.

[49] M.I. Lopez, P.E. Meza Martinez, M.A. Meyers, Acta Biomater, 10 (2014) 2056-2064.

[50] R.S. Rivlin, A.G. Thomas, Philos Tr R Soc S-A, 243 (1951) 289-298.

[51] V.A. Lubarda, J Mech Phys Solids, 58 (2010) 860-873. 

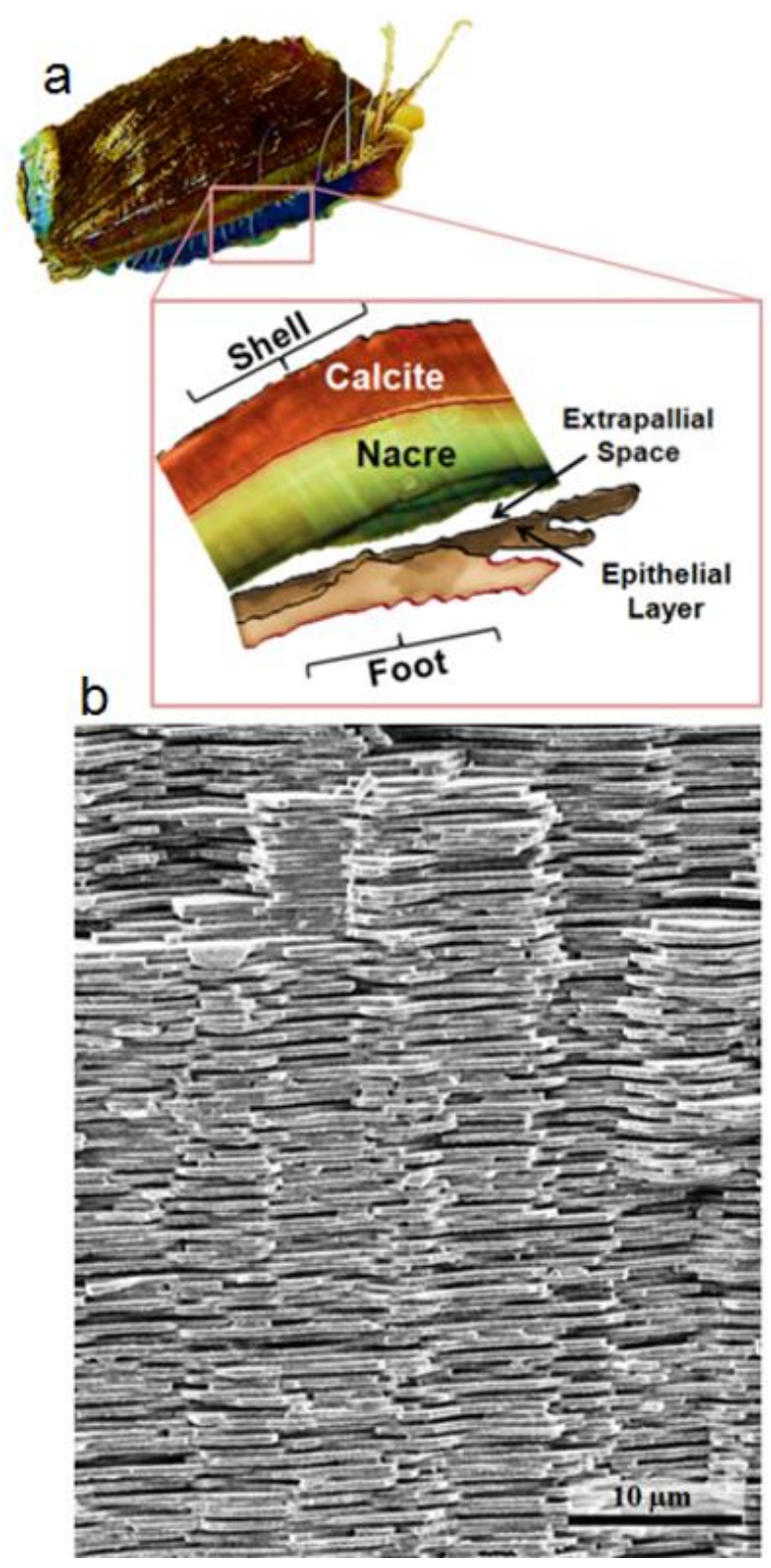

Figure 1: a) Structure of abalone shell showing the calcitic and nacreous layer that is in direct contact with the epithelial layer. The epithelial layer is the inner part of the abalone foot. b) Fracture surface in red abalone nacre. Nanoscale tiles of aragonite separated by an organic interlamellar layers of organic biopolymers, described as "brick-and mortar" structure. The regular stacking of tablets with interdigitation can be seen. 


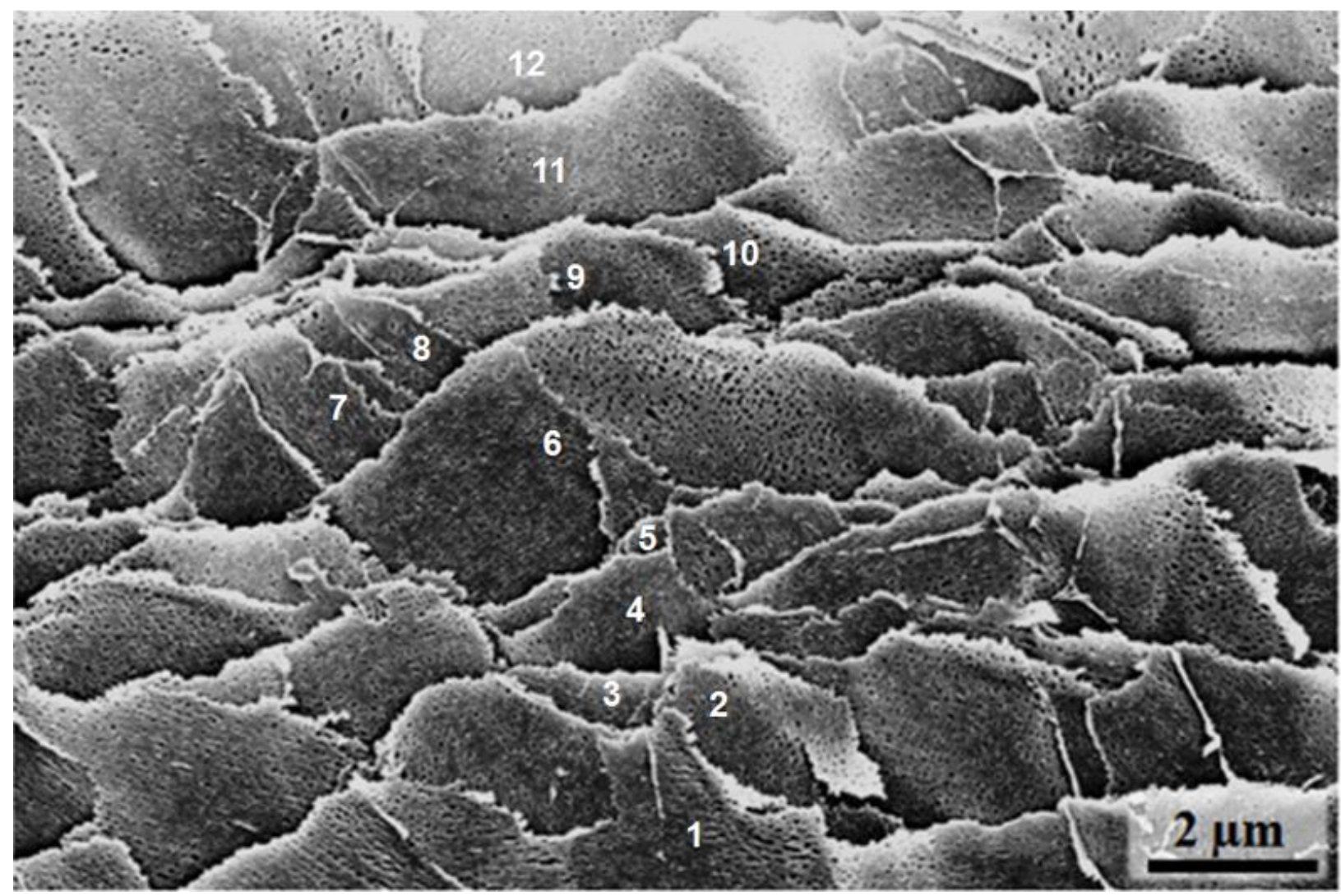

Figure 2: Stacking of organic interlamellar (intertabular) layers of demineralized nacre; 12 layers marked. 

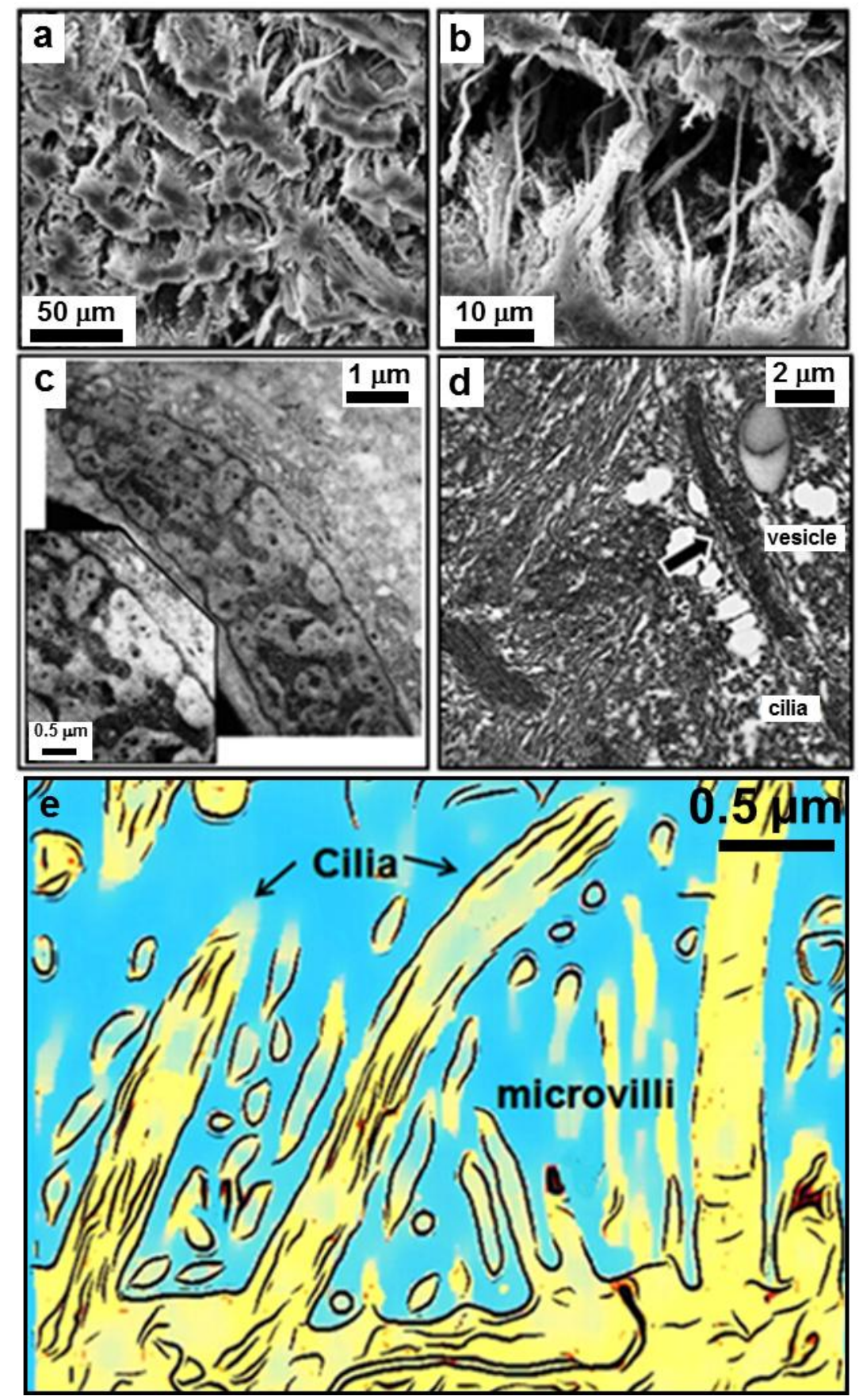

Figure 3: a) SEM micrograph of the surface of the mantle epithelium showing the cilia (hair-like protrusions). b) High resolution of (a). c) Transmission electron micrograph showing a secretory cell; boundary marked by arrow and detail showing the pigmented granules. d) TEM micrograph of epithelial mantle showing cilia and a mucous vesicle (marked by arrow) with electron lucent secretory product. e) Schematic rendition showing microvilli and cilia. 


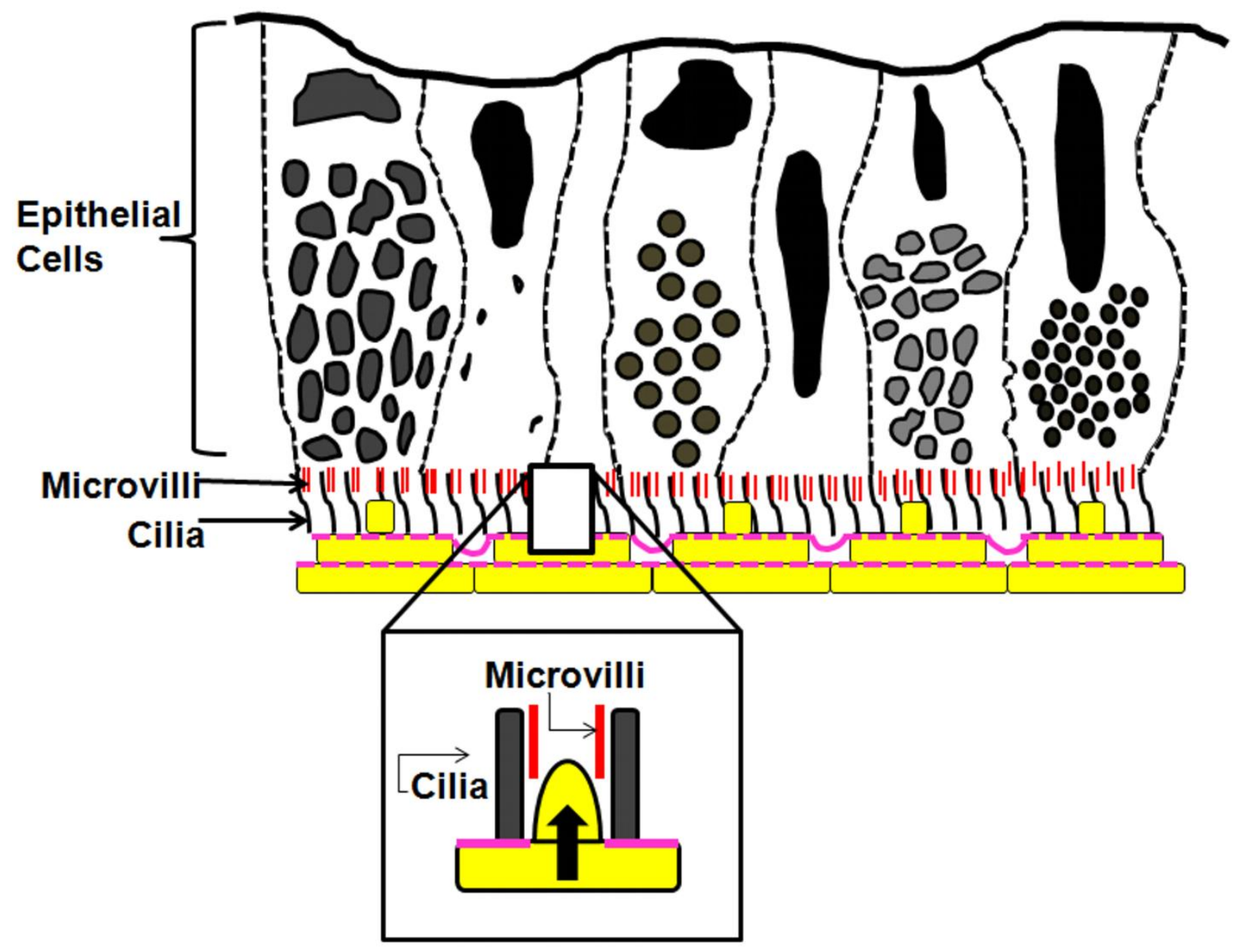

Figure 4: Schematic depiction of the epithelium cells containing cilia and microvilli which are involved in the deposition and positioning of the organic membrane composed of chitin fibrils and acidic molecules. 


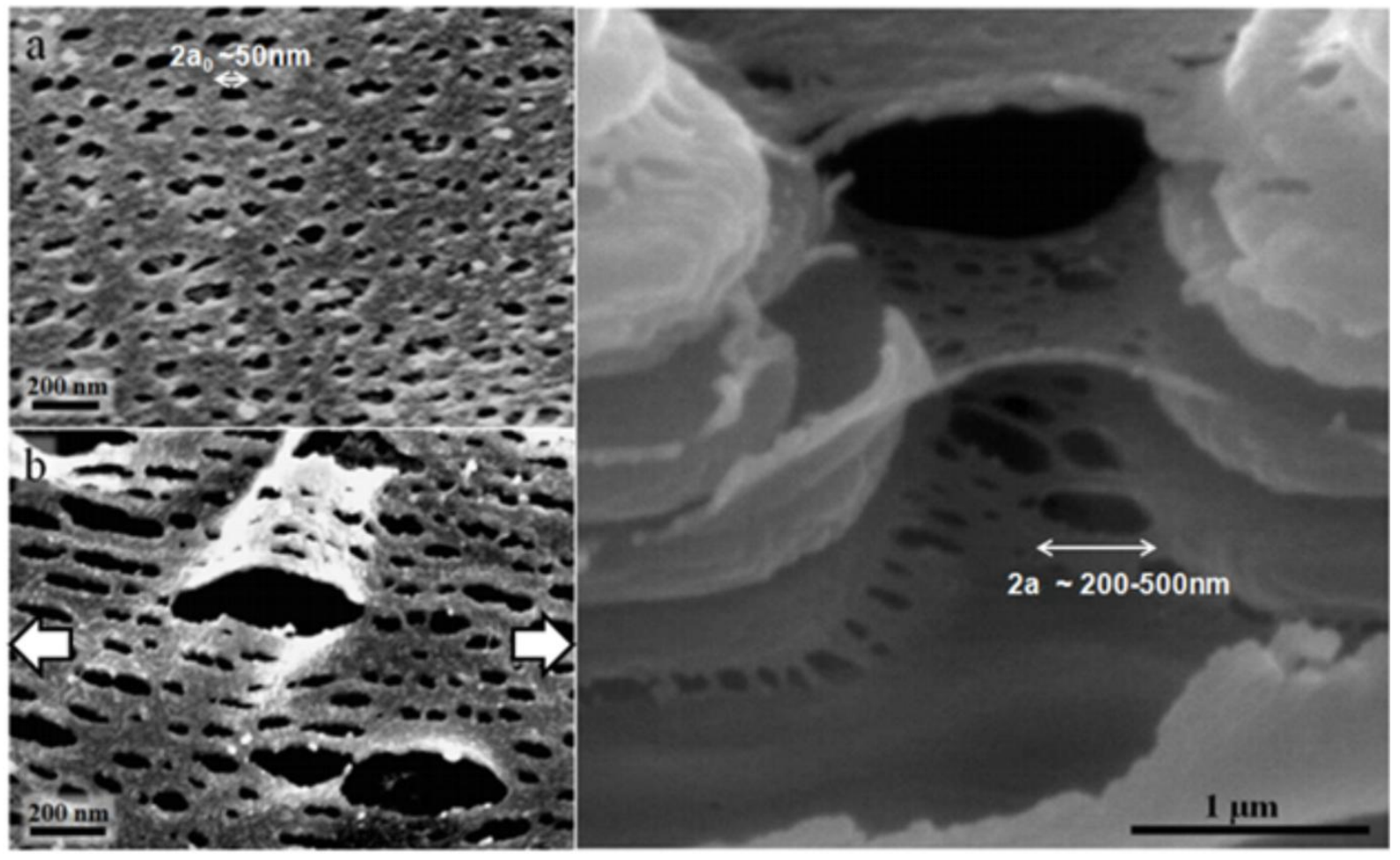

Figure 5: a) Pores (unstretched). b) Pores stretched uniaxially. c) Pores stretched due to the separation of the tablet stacks and/or weight of the membrane. 


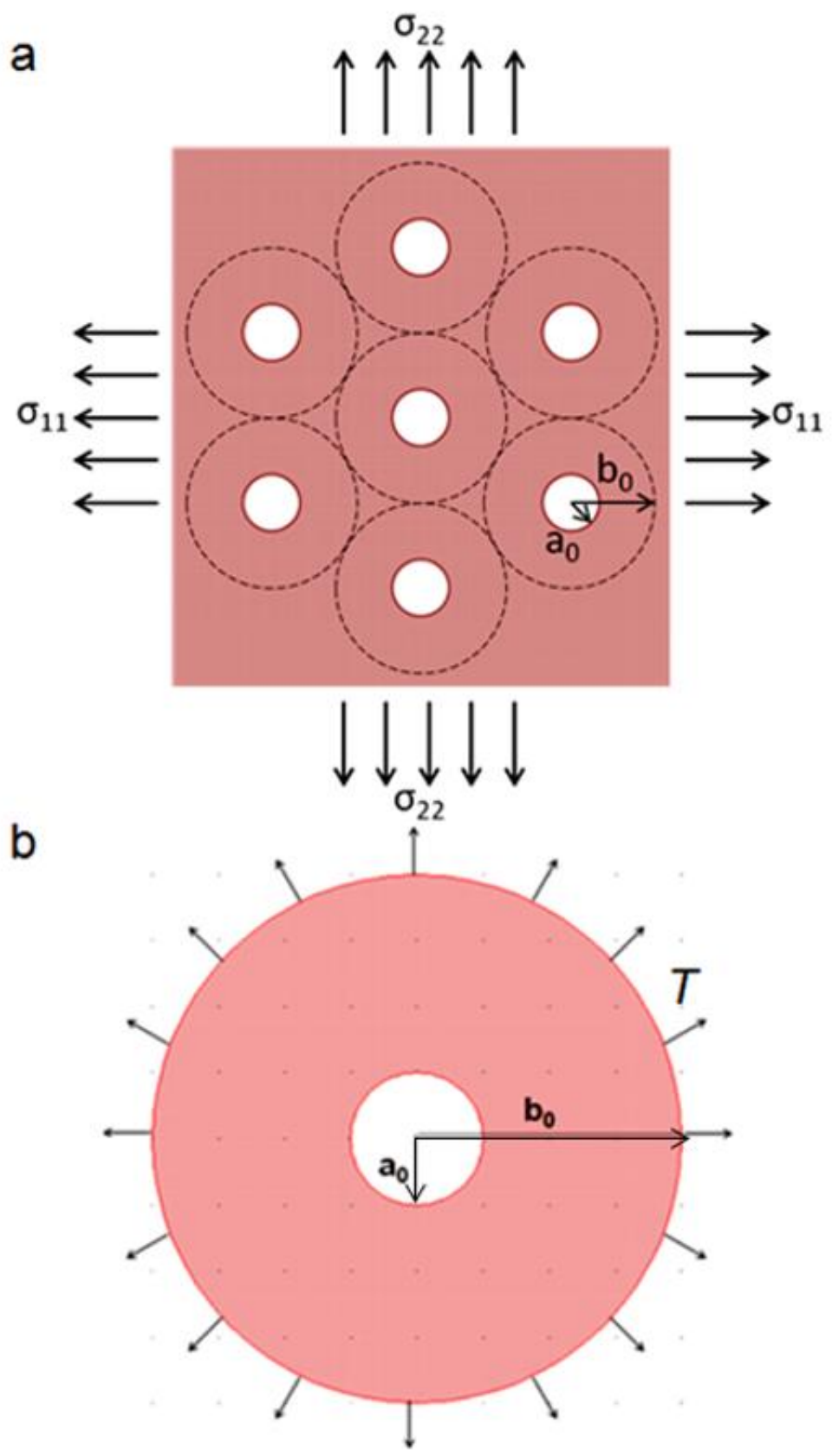

Figure 6: a) Idealized arrangement of holes subject to biaxial loading. b) Separate representative areal element of membrane with pore. 


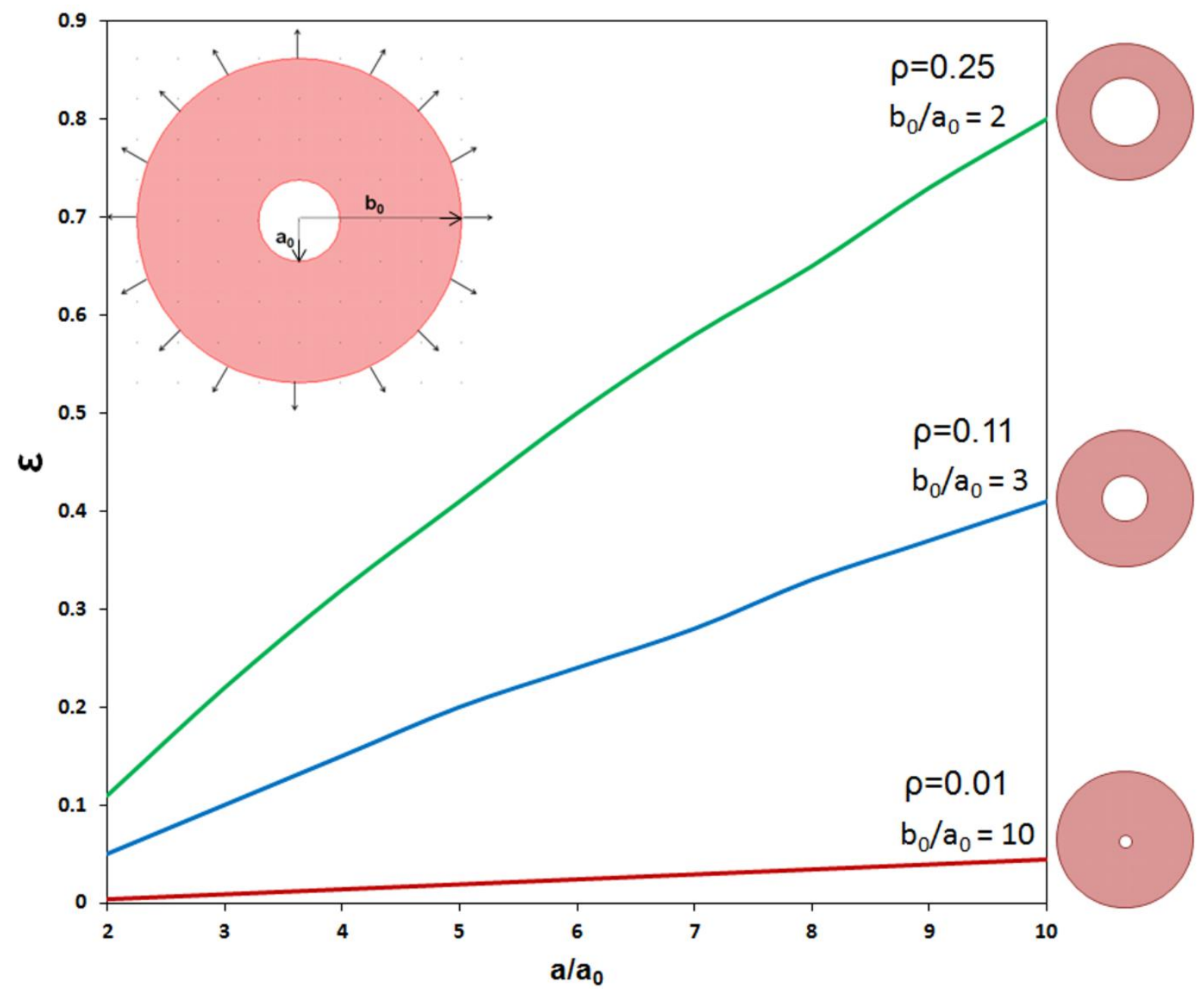

Figure 7: Strain values achieved compared to the relative pore to membrane size. Increase in normalized pore radius $\left(a / a_{0}\right)$ with global strain for different values of porosity ( $\rho$, defined as

$-\quad)$. For $-\quad, a$ is equal to $10 a_{0}$ for a strain of $\varepsilon=0.03$. 

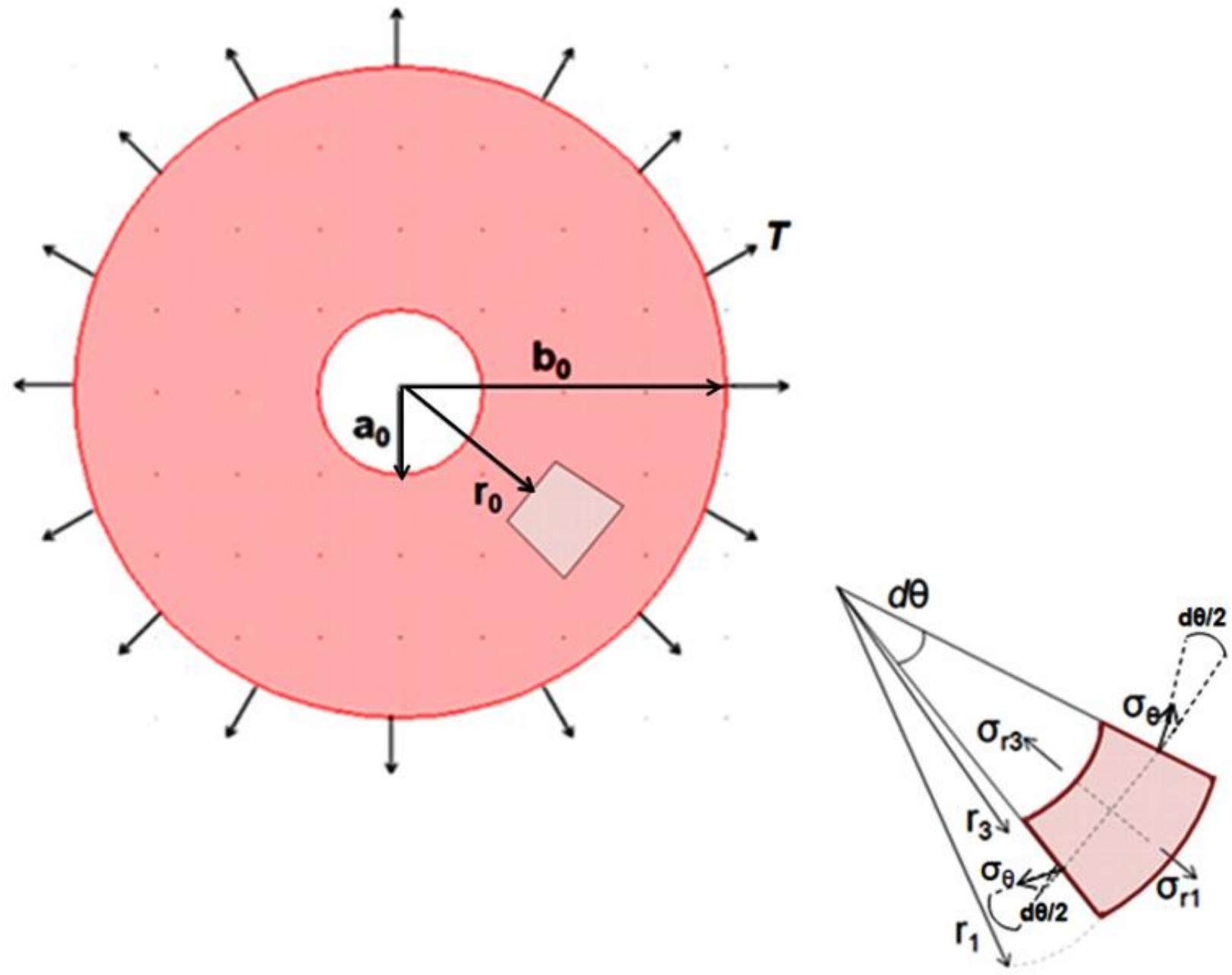

Figure 8: Areal element subjected to radial and hoop stresses in membrane containing circular pore and subjected to external traction $T$. 


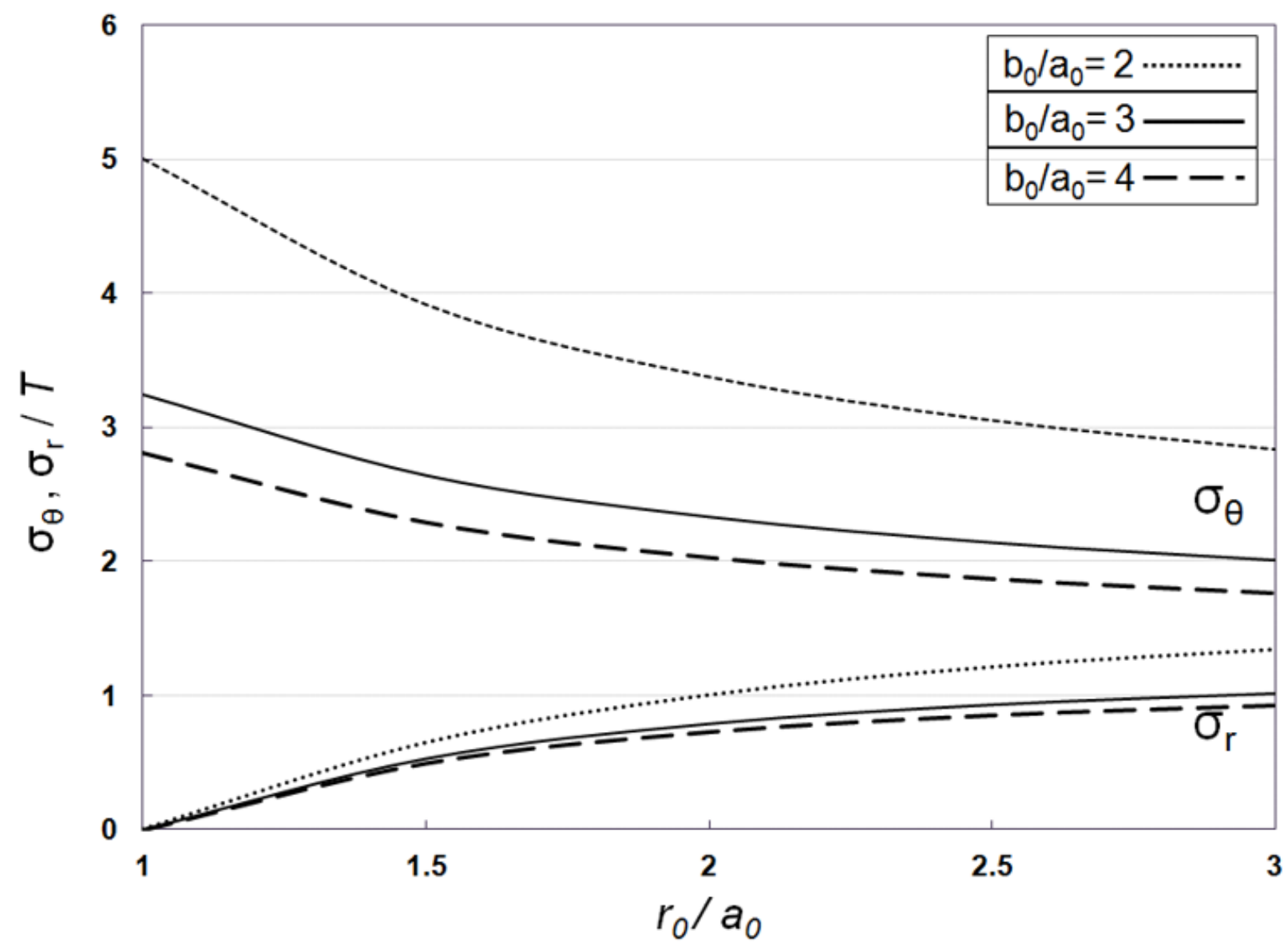

Figure 9: Variation of radial and hoop stresses (normalized by $T$ ) with normalized radius for three ratios $-2,3$, and 4. Applied traction $T / \mu=0.5$. 


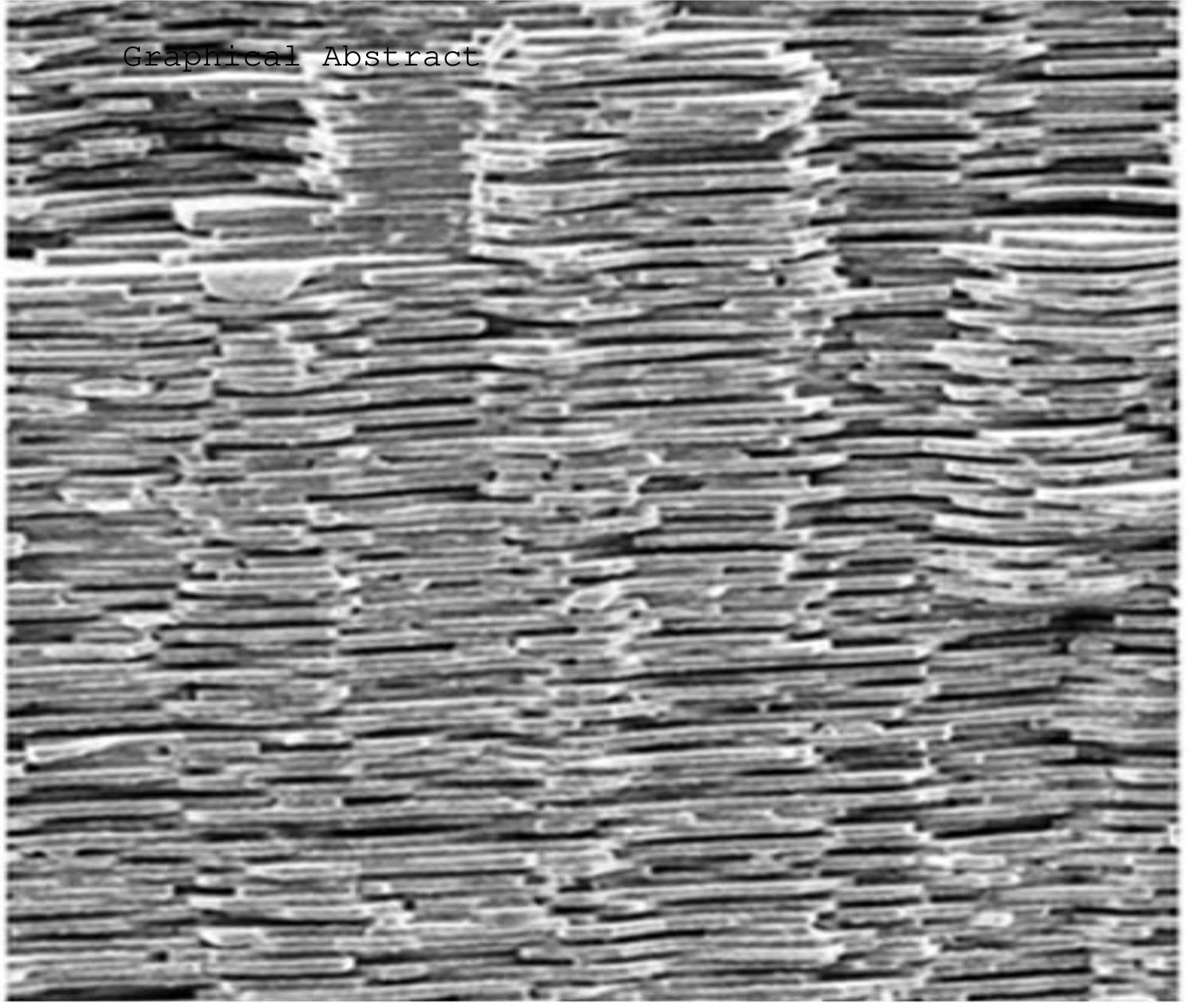

